\title{
Effects of fatigue on trunk stability in elite gymnasts
}

\author{
Jaap H. van Dieën $\cdot$ Tessy Luger · Jeroen van der Eb
}

Received: 6 April 2011/ Accepted: 7 July 2011/Published online: 23 July 2011

(c) The Author(s) 2011. This article is published with open access at Springerlink.com

\begin{abstract}
The aim of the present study was to test the hypothesis that fatigue due to exercises performed in training leads to a decrement of trunk stability in elite, female gymnasts. Nine female gymnasts participated in the study. To fatigue trunk muscles, four series of five dump handstands on the uneven bar were performed. Before and after the fatigue protocol, participants performed three trials of a balancing task while sitting on a seat fixed over a hemisphere to create an unstable surface. A force plate tracked the location of the center of pressure (CoP). In addition, nine trials were performed in which the seat was backward inclined over a set angle and suddenly released after which the subject had to regain balance. Sway amplitude and frequency in unperturbed sitting were determined from the CoP time series and averaged over trials. The maximum displacement and rate of recovery of the CoP location after the sudden release were determined and averaged over trials. After the fatigue protocol, sway amplitude in the fore-aft direction was significantly increased $(p=0.03)$, while sway frequency was decreased ( $p=0.005)$. In addition, the maximum displacement after the sudden release was increased $(p=0.009)$, while the rate of recovery after the perturbation was decreased
\end{abstract}

Communicated by Toshio Moritani.

This project was approved by the IRB of the Faculty of Human Movement Sciences, VU University Amsterdam, The Netherlands, number 2009-039.

J. H. van Dieën $(\varangle) \cdot$ T. Luger · J. van der Eb

Faculty of Human Movement Sciences,

Research Institute MOVE, VU University Amsterdam,

Van der Boechorststraat 9, 1081 BT Amsterdam,

The Netherlands

e-mail: j.vandieen@fbw.vu.nl $(p=0.05)$. Fatigue induced by series of exercises representing a realistic training load caused a measurable decrement in dynamic stability of the trunk in elite gymnasts.

Keywords Core stability - Balance $\cdot$ Localized muscle fatigue

\section{Introduction}

Stability of the trunk, often referred to as core stability, has gained considerable attention in the recent years. It can be operationally defined as: "the body's ability to control the trunk in response to internal and external disturbances, including the forces generated from distal body segments as well as from expected or unexpected perturbations" (Zazulak et al. 2007). Core stability training has become a major element of training programs in sports as well as rehabilitation (Borghuis et al. 2008). It seems plausible that core stability is important for injury prevention and athletic performance, especially in sports with large demands for balance control, such as gymnastics.

The importance of core stability for injury prevention in athletes has received some support from epidemiological studies relating low core stability to incidence of injuries of the back (Cholewicki et al. 2005) and lower extremities (Zazulak et al. 2007; Leetun et al. 2004). In addition, there is limited evidence to support its role as a determinant of performance. Nesser et al. found a moderate correlation between core stability and performance in football players (Nesser et al. 2008). It should be noted that, in several of the studies mentioned (Nesser et al. 2008; Leetun et al. 2004), the measurements used to determine core stability reflect muscle strength and endurance, possible determinants of stability, rather than stability itself. 
The stability of the spine and trunk depends on contributions of the passive, active and control sub-systems (Panjabi 1992). The passive joint structures such as ligaments and intervertebral discs contribute to stability by providing joint stiffness (Cholewicki et al. 1997). The active sub-system, the trunk musculature contributes by further increasing stiffness through cocontraction (Cholewicki et al. 1997; Gardner-Morse and Stokes 1998; Dieën et al. 2003; Stokes and Gardner-Morse 2003). In addition, the control sub-system, i.e., the nervous system, controls reflexive and triggered muscle activity contributing to stability (Moorhouse and Granata 2007), based on sensory feedback from muscle and joint receptors, and feedback from the visual and vestibular systems (Goodworth and Peterka 2009).

A model study predicted that the decrease in muscle stiffness that is associated with fatigue (Kirsch and Rymer 1987; Zhang and Rymer 2001), may impair trunk stability (Granata et al. 2004). Given the importance of reflexive muscle activity for core stability, the reductions in maximum force and rate of force rise that develop with muscle fatigue can also be expected to impair core stability, which may be aggravated by the adverse effects of fatigue on proprioception (Taimela et al. 1999) and force steadiness (Missenard et al. 2008).

Previous studies showed that trunk muscle fatigue impaired balance control (Davidson et al. 2004, 2009), which was attributed to a reduced control over the trunk. Granata and Gottipati (2008) showed that fatigue of the extensor muscles had a negative effect on the trunk's local dynamic stability, i.e., the responses to the small perturbations that are always present due to neuromuscular noise (Dieën et al. 2008). Herrmann et al. found decreased contact forces during an external perturbation of the trunk induced with a swinging pendulum, which indicates reduced trunk stiffness (Herrmann et al. 2006). Using a similar paradigm, Dupeyron et al. (2010), however, found no change in contact forces. In both of these studies, increased electromyographic amplitudes of the reflex responses indicated compensatory mechanisms to counteract the fatigue effects. Moreover, several studies found increased cocontraction in unperturbed standing after inducing trunk muscle fatigue, as a potential compensatory mechanism (Grondin and Potvin 2009; Granata et al. 2001, 2004; Allison and Henry 2002). Two of these studies also investigated the response to an external perturbation and found no effects of fatigue (Grondin and Potvin 2009; Granata et al. 2004), suggesting that such compensations were adequate.

The evidence for the effects of fatigue on trunk stability from the studies reviewed above is not consistent. Furthermore, all of these studies involved high fatigue levels (typically a reduction in force producing capacity by $40 \%$ ) induced using isolated, non-functional activity of trunk muscles. Also subject populations were non-athletes. It thus remains unclear whether fatigue of the trunk muscles that even well-trained athletes may develop during training or competitive events can induce impairments of trunk stability. The aim of the present study therefore was to investigate the effects of fatigue induced by a set of exercises as performed regularly in training on trunk stability in elite gymnasts. We hypothesized that gymnasts show increased sway amplitudes in a seated balancing task and that they are less able to correct an external perturbation of seated balance after a set of gymnastic exercises.

\section{Methods}

Nine gymnasts, all girls, with a mean age of 12.4 $( \pm 2.3)$ years old participated in the study. Subjects were recruited through the gymnast association 'Flik Flak' 's Hertogenbosch, The Netherlands. Participants performed at the national top level. Their mean ( \pm standard deviation) height and body mass were $1.47 \pm 0.12 \mathrm{~m}$ and $39.0 \pm 12.92 \mathrm{~kg}$, respectively. None of the participants reported any recent history of injuries that did not allow training participation. All gymnasts and their parents provided informed consent before participation.

The experimental protocol, which had been approved by the ethical committee of the Faculty of Human Movement Sciences of the VU University Amsterdam (number 2009-039), comprised a 10-min fatigue protocol in between the pre- and posttests of trunk stability. The measurements were performed at the beginning of regular training sessions in the afternoon, after school hours. Prior activities, either during the day before the training or during warming-up were not controlled. The fatigue protocol contained four series of five dump handstand exercises on the uneven bar. Based on subjective report of trainers and gymnasts, this bout of exercises was expected to fatigue the trunk flexor and extensor muscles. Series of dump handstands are a regular part of normal training and the intensity of this bout of exercises was comparable to the more intensive elements of the participant's normal training activities

For the measurements of trunk stability, subjects were seated with arms in their lap on an unstable seat, which required them to dynamically balance by trunk movement only (Fig. 1). The seat was mounted over a hemisphere (radius of hemisphere: $25 \mathrm{~cm}$, height of the seat relative to the lowest point on the hemisphere: $17 \mathrm{~cm}$ ), creating instability in all directions. To trace the center of pressure $(\mathrm{CoP})$, the seat was placed on a custom-made strain gauge force plate that was sampled at 200 samples/s. The force plate was calibrated prior to each measurement session, by placing known weights on the plate. Foot supports were 
Fig. 1 Schematic of the unperturbed seated balancing task (a) and the sudden release task (b) a

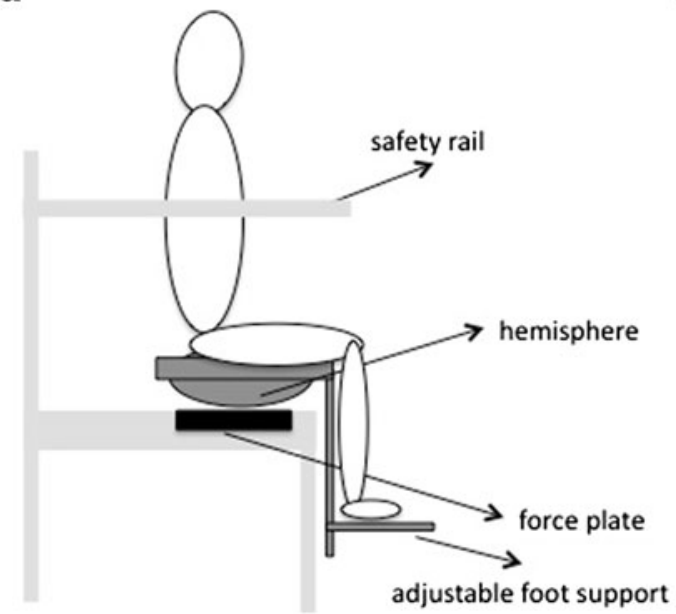

b

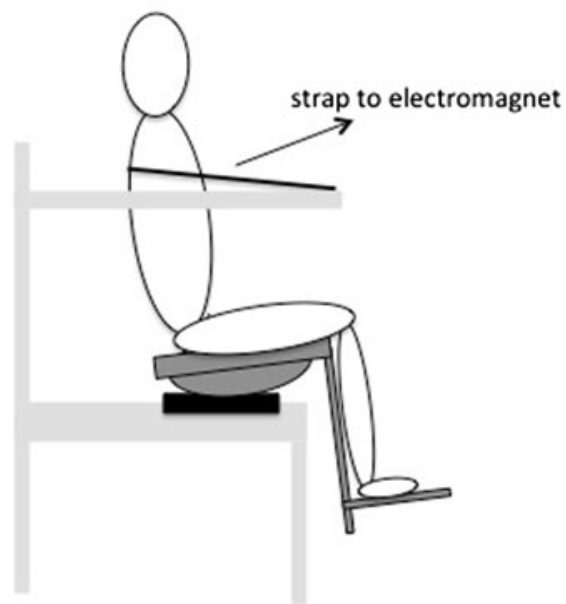

attached to the seat to prevent influence of leg movements. The footplate was adjusted to support the feet with the knees and hips at $90^{\circ}$ angles. A rail was built around the seat for safety. Trunk balance was tested using two tasks. The first task required the subject to sit as still as possible and lasted $30 \mathrm{~s}$. Three repetitions of this task were performed before and after the fatiguing exercise, as reliability of single measurements was previously shown to be poor (Dieën et al. 2010b). In the second task, the subject leaned back on the seat, supported by a strap around the thorax that was attached to two electromagnets on the safety rail in front of the subject. The length of the strap was adjusted to obtain a constant inclination angle for each subject. After a random interval of 3-7 s after the start of the measurement, the electromagnets were released and the subject had to regain balance as quickly as possible. Recording of data was continued for a total of $20 \mathrm{~s}$. Nine repetitions were performed within a few minutes before and after the fatigue exercise.

Force plate data were low-pass filtered at $10 \mathrm{~Hz}$ (fourth order Butterworth), as the signals contained only very limited power above $3 \mathrm{~Hz}$, while sensitivity analysis showed only minor effects of filtering at either a higher $(20 \mathrm{~Hz})$ or lower $(2.5 \mathrm{~Hz})$ cutoff. Subsequently, CoP positions were calculated. For the unperturbed seated task, four independent parameters were derived from the CoP time series of the 5th till 30th second of the trial: the RMS of the CoP in $x$ and $y$ direction $\left(\mathrm{RMS}_{x}\right.$, left-right; $\mathrm{RMS}_{y}$, antero-posterior) and the mean power frequency of the $\mathrm{CoP}$ time series in both directions $\left(\mathrm{MPF}_{x}\right.$ and $\left.\mathrm{MPF}_{y}\right)$.

For the unperturbed trials (see Fig. 2 for an example), the mean $\mathrm{CoP}$ position determined over the first $2 \mathrm{~s}$ was first subtracted from the time series. Next, the maximum $\mathrm{CoP}$ position in the $y$ direction was determined, which reflects the maximum backward $\mathrm{CoP}$ displacement after the sudden release $\left(\mathrm{MAX}_{y}\right)$. Subsequently, an exponential decay function was fitted to the CoP time series in the $y$ direction from the instant of $\mathrm{MAX}_{y}$ and the subsequent 7 s (Fig. 3; Bruijn et al. 2010):

$Y(t)=Y^{\prime}+\left(\operatorname{MAX}_{y}-Y^{\prime}\right) \times e^{-\lambda t}$

with time $t$ defined as zero at the instant of $\operatorname{MAX}_{y}$, $Y$ referring to the $y$ coordinate of $\mathrm{CoP}, Y^{\prime}$ referring to the steady state $y$ position of the CoP to which the subject converged, estimated as the median position over the fifth till seventh second after the maximum backward displacement, and $\mathrm{MAX}_{y}$ referring to the maximum displacement. The parameter $\lambda$ was used as an indicator of the rate of recovery after the perturbation, with higher values indicating faster recovery.

For all six dependent variables, median values over the repeated trials per subject were used for statistical analysis to avoid effects of trials with outlying results. Comparisons between the fatigued and unfatigued conditions were made based on averages over subjects, which were tested with paired $t$ tests, with $\alpha=0.05$. The effect of trial order was tested over the trials performed before the fatiguing exercise using repeated measures ANOVA.

\section{Results}

Figure 2 illustrates a typical example of the data obtained in the unperturbed trials. Substantial variation of both RMS and MPF values was apparent between the trials. However, the $\mathrm{RMS}_{y}$ values were on average higher and $\mathrm{MPF}_{y}$ values were on average lower after than before the fatiguing exercise, whereas no difference was discernible in the $x$ direction. The effects in the $y$ direction (anterior-posterior) were in line with our hypothesis and were found to 
Fig. 2 Typical examples of: the CoP time series in the unperturbed trials with anteriorposterior displacement in black and left-right displacement in gray (a), the spatial distribution of the CoP position in the same trial (b), the $\mathrm{RMS}_{x}$ (black) and RMSy (gray) values for all trials of the same subject (c), the $\mathrm{MPF}_{x}$ (black) and $\mathrm{MPF}_{y}$ (gray) values for all trials of the same subject (d). The fatiguing exercise was performed between trials three and four a

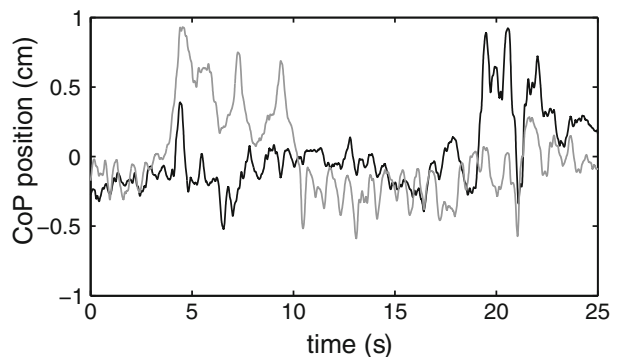

C

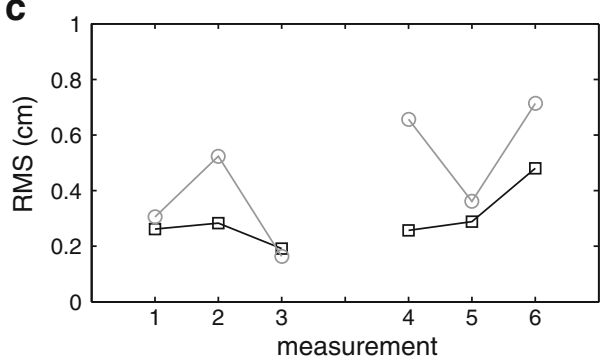

a

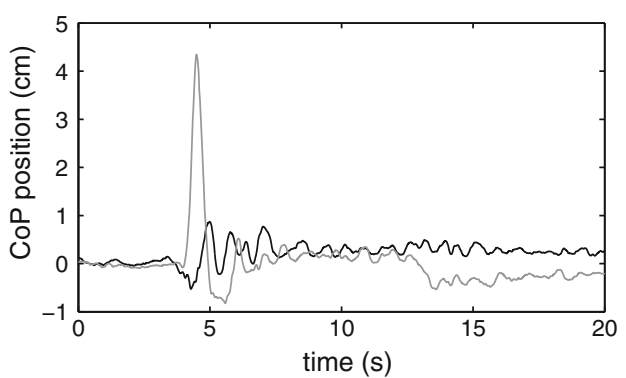

C

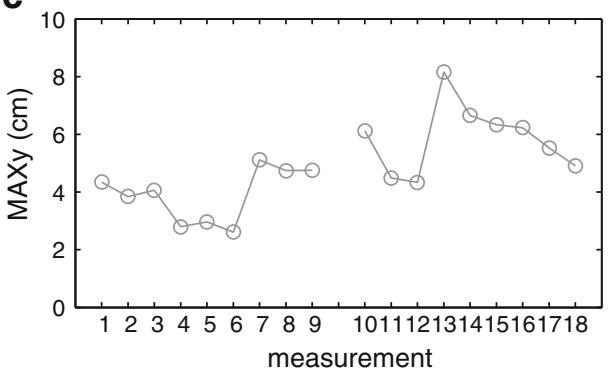

b

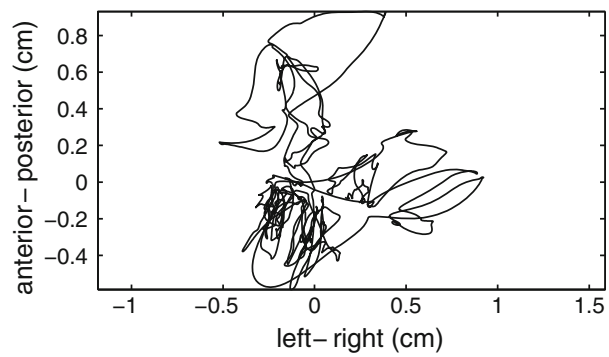

d

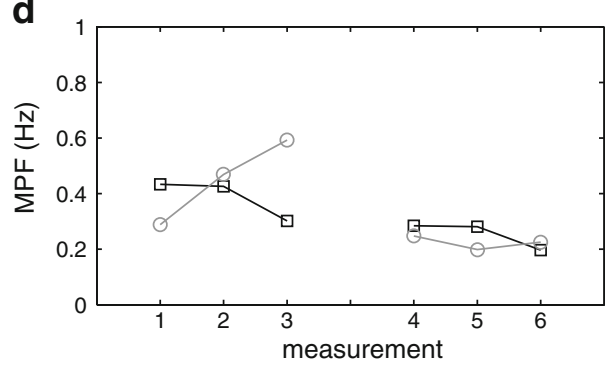

b

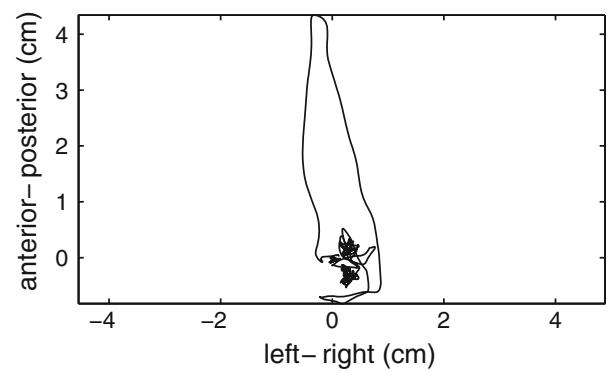

d

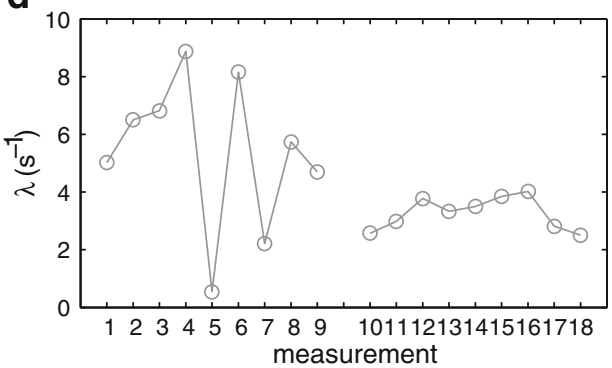

be statistically significant for the group (Table 1). No effects were found in the $x$ direction.

Figure 3 illustrates a typical example of the CoP data in the perturbed trials. After the sudden release approximately $4 \mathrm{~s}$ after the start of the trial, the subject moved further backward to recover and attain a fairly steady position within $3 \mathrm{~s}$ after the release. Again, substantial variation of the parameter values was apparent between the trials. However, the $\operatorname{MAX}_{y}$ values were on average higher and $\lambda$ values were on average lower after than before the fatiguing exercise. These effects were in line with our hypothesis and were found to be statistically significant for the group (Table 1).
To test whether the differences between the trials before and after the fatiguing exercise could be explained by order effects, we tested for the effect of measurement order over the trials collected before the fatiguing exercise. None of the variables showed a significant order effect $(p>0.383)$.

\section{Discussion}

This study was designed to test the hypotheses that gymnasts show increased sway amplitudes in a seated balancing task and that they are less able to correct an external perturbation of seated balance after a set of fatiguing 
Table 1 Means and standard deviations of all dependent variables before and after the bout of fatiguing exercise and $p$ values for differences between the fatigued and unfatigued conditions based on paired $t$ tests

\begin{tabular}{lll}
\hline Independent variable & Mean $\pm \mathrm{SD}$ & $p$ \\
\hline $\mathrm{RMS}_{x}(\mathrm{~cm})$ & & \\
Unfatigued & $0.19 \pm 0.08$ & 0.549 \\
Fatigued & $0.21 \pm 0.09$ & \\
$\mathrm{RMS}_{y}(\mathrm{~cm})$ & & 0.030 \\
Unfatigued & $0.18 \pm 0.08$ & \\
Fatigued & $0.31 \pm 0.17$ & \\
$\mathrm{MPF}_{x}(\mathrm{~Hz})$ & & 0.810 \\
Unfatigued & $0.40 \pm 0.15$ & \\
Fatigued & $0.41 \pm 0.24$ & 0.005 \\
$\mathrm{MPF}_{y}(\mathrm{~Hz})$ & & \\
Unfatigued & $0.52 \pm 0.13$ & \\
Fatigued & $0.35 \pm 0.17$ & \\
$\mathrm{MAX}_{y}$ (cm) & & 0.003 \\
Unfatigued & $3.81 \pm 0.90$ & \\
Fatigued & $5.10 \pm 1.00$ & \\
$\lambda$ (s ${ }^{-1}$ ) & & \\
Unfatigued & $1.73 \pm 0.58$ & \\
Fatigued & $0.80 \pm 0.27$ & \\
\hline
\end{tabular}

gymnastic exercises. As hypothesized, unperturbed seated balancing performance was decreased in the anterior-posterior direction as evidenced by a larger amplitude and lower frequency. However, no effect was found on sway in the frontal plane. Perturbations were performed in the posterior direction only. In line with the hypothesis, the maximum displacement after the perturbation was larger and the recovery of balance was slower after the exercise than before.

It is at present unclear why effects were found in the sagittal plane (anterior-posterior direction) only. An explanation could be that the dump hand stands fatigued the abdominal muscles selectively given the mechanics of these exercises. While abdominal muscles can contribute to control of lateral bending of the trunk (Seroussi and Pope 1987), the extensor muscles have a higher moment producing capacity in the frontal plane (Dieën and Kingma 1999) and probably allow more precise control in view of their abundant insertions on the multi-segmented lumbar spine. Thus, while control in frontal plane could be done mainly by the extensor musculature, the abdominal muscles are obviously indispensible for control in the sagittal plane. Hence, selective fatigue of these muscles might explain why effects were restricted to the sagittal plane.

As reported in the introduction, the previous studies on the effects of fatigue on trunk stability showed inconsistent effects. Most of these tested trunk stability by applying perturbations to the trunk in either standing or sitting positions. It was suggested that compensatory mechanisms such as increased cocontraction and increased reflex gains could explain the absence of fatigue effects in some of these studies. In the present study, we used a dynamic balancing task to test trunk stability. In this task, the center of mass unavoidably sways beyond the surface of support, which is the point of contact of the hemisphere on the force plate. Trunk moments, accelerating the upper body relative to the pelvis and seat, must then be used to bring the center of mass back over the surface of support, while stiffening the trunk would not be effective (cf Otten 1999). It has previously been shown that cocontraction, which would stiffen the trunk is not effective in this task (Reeves et al. 2006). The present task may therefore be more sensitive to trunk muscle fatigue than tasks in which the support surface is larger. Activity of the trunk muscles was not measured and it is therefore unknown whether subjects increased cocontraction, but this would be consistent with the increase in $\mathrm{RMS}_{y}$ (Reeves et al. 2006) and decrease in $\mathrm{MPF}_{y}$ (Dieën et al. 2010a).

Several mechanisms could underlie the fatigue effects observed. With fatigue of an agonistic muscle, the activation levels need to be increased to maintain force output. This increases unsteadiness of muscle force (Missenard et al. 2009), which would cause increased sway and thus could explain the increase in $\mathrm{RMS}_{y}$. Variability of muscle force would increase even more when antagonistic cocontraction would be increased (Selen et al. 2005) which, given the fact that stiffening the trunk does not limit kinematic variability in the present task, could further increase sway amplitudes. Fatigue will likely slow down muscular responses due to increased proprioceptive thresholds (Taimela et al. 1999) and due to the slower force development in the fatigued muscles (De Ruiter et al. 1999; Perrey et al. 2010). Slower responses to balance perturbation would increase sway amplitude (Radebold et al. 2001) and likely decrease sway frequency in the unperturbed task and would also increase the amplitude and reduce the recovery in the perturbed task (Reeves et al. 2009). Increased proprioceptive thresholds might be reflected specifically in an increase in the maximum displacement after the perturbation (increased $\mathrm{MAX}_{y}$ ), while a decreased rate of force development might be more obvious in a decreased rate of recovery (decreased $\lambda$ ). Finally, fatigue could be associated with an increased respiratory challenge, which might affect trunk stability (Janssens et al. 2010). It is to be expected that the respiratory effect would be most obvious in the sagittal plane, which would be in line with the fact that only effects in the $y$ direction were found.

Unavoidably, trials in the fatigued condition were performed after those in the unfatigued condition and this could lead to order effects, for example due to learning, which might dilute the fatigue effect. The absence of an 
order effect over the trials performed before the fatiguing exercise suggests that such effects did not occur, possibly due to the fact that the participants were all highly trained athletes.

It is unclear to what extent the loss of trunk stability as found in the present study would affect the performance of gymnastic activities. However, in many gymnastics exercises, balance and trunk stability are severely challenged. Furthermore, impaired trunk stability may limit performance and increase injury risk. Therefore, the fact that trunk stability was negatively affected by a series of fatiguing exercises that reflect intensity of typical training activities and competitive events suggests several practical implications. The results suggest that trainers should take fatigue effects into account when planning the order of training activities, e.g., avoid balance beam exercises after uneven bar or horizontal bar exercises. Furthermore, the results may have implications for the intensity of warming up exercises for the competitive events. Finally, the results indicate that endurance training of trunk muscles and perhaps balance training may need to be performed not only in rested, but also in fatigued condition.

\section{Conclusion}

In conclusion, the present study showed that trunk stability in elite gymnasts was negatively affected by a bout of exercises, which reflected normal training activities. Both sagittal plane sway in unperturbed balancing and recovery after a backward balance perturbation were affected. These results suggest that fatigue effects on trunk stability should be taken into account in the planning and design of gymnastics training.

Open Access This article is distributed under the terms of the Creative Commons Attribution Noncommercial License which permits any noncommercial use, distribution, and reproduction in any medium, provided the original author(s) and source are credited.

\section{References}

Allison GT, Henry SM (2002) The influence of fatigue on trunk muscle responses to sudden arm movements, a pilot study. Clin Biomech 17:414-417

Borghuis J, Hof AL, Lemmink KAPM (2008) The importance of sensory-motor control in providing core stability: implications for measurement and training. Sports Med 38:893-916

Bruijn SM, Meijer OG, Beek PJ, JHv Dieën (2010) The effects of arm swing on human gait stability. Exp Biol 213:3945-3952

Cholewicki J, Panjabi MM, Khatchatryan A (1997) Stabilizing function of trunk flexor/extensor muscles around a neutral spine posture. Spine 22:2207-2212

Cholewicki J, Silfies SP, Shah RA, Greene HS, Reeves NP, Alvi K, Goldberg B (2005) Delayed trunk muscle reflex responses increase the risk of low back injuries. Spine 30:2614-2620
Davidson BS, Madigan ML, Nussbaum MA (2004) Effects of lumbar extensor fatigue and fatigue rate on postural sway. Eur J Appl Physiol 93:183-189

Davidson BS, Madigan ML, Nussbaum MA, Wojcik LA (2009) Effects of localized muscle fatigue on recovery from a postural perturbation without stepping. Gait Posture 29:552-557

De Ruiter CJ, Jones DA, Sargeant AJ, De Haan A (1999) Temperature effect on the rates of isometric force development and relaxation in the fresh and fatigued human adductor pollicis muscle. Exp Physiol 84:1137-1150

Dieën JHv, Kingma I (1999) Total trunk muscle force and spinal compression are lower in asymmetric moments as compared to pure extension moments. J Biomech 32:655-662

Dieën JHv, Kingma I, Burg JCEvd (2003) Evidence for a role of antagonistic cocontraction in controlling trunk stiffness during lifting. J Biomech 36:1829-1836

Dieën JHv, Selen LPJ, Beek PJ (2008) Suppression of neuromuscular noise through impedance modulation. In: de Jong DA (ed) Progress in biological cybernetics research. Nova Publishers, New York, pp 137-160

Dieën JHv, Koppes L, Twisk J (2010a) Low-back pain history and postural sway in unstable sitting. Spine 35:812-817

Dieën JHv, Koppes L, Twisk J (2010b) Postural sway parameters in seated balancing; their reliability and relationship with balancing performance. Gait Posture 31:42-46

Dupeyron A, Perrey S, Micallef JP, Pellisier J (2010) Influence of back muscle fatigue on lumbar reflex adaptation during sudden external force perturbations. $\mathrm{J}$ Electromyogr Kinesiol 20:426-432

Gardner-Morse MG, Stokes IA (1998) The effects of abdominal muscle coactivation on lumbar spine stability. Spine 23:86-91

Goodworth AD, Peterka RJ (2009) Contribution of sensorimotor integration to spinal stabilization in humans. J Neurophysiol 102:496-512

Granata KP, Gottipati P (2008) Fatigue influences the dynamic stability of the torso. Appl Ergonomics 51:1258-1271

Granata KP, Orishimo KF, Sanford AH (2001) Trunk muscle coactivation in preparation for sudden load. J Electromyogr Kinesiol 11:247-254

Granata KP, Slota GP, Wilson SE (2004) Influence of fatigue in neuromuscular control of spinal stability. Hum Factors 46:81-91

Grondin DE, Potvin JR (2009) Effects of trunk muscle fatigue and load timing on spinal responses during sudden hand loading. J Electromyogr Kinesiol 19:e237-e245

Herrmann CM, Madigan ML, Davidson BS, Granata KP (2006) Effect of lumbar extensor fatigue on paraspinal muscle reflexes. J Electromyogr Kinesiol 16:637-641

Janssens L, Brumagne S, Polspoel K, Troosters T, McConnell A (2010) The effect of inspiratory muscles fatigue on postural control in people with and without recurrent low back pain. Spine 35:1088-1094

Kirsch RF, Rymer WZ (1987) Neural compensation for muscular fatigue: evidence for significant force regulation in man. J Neurophysiol 57:1893-1910

Leetun DT, Lloyd Ireland M, Wilson JD, Ballantyne BT, McClay Davis I (2004) Core stability measures as risk factors for lower extremity injury in athletes. Med Sci Sports Exerc 36:926-934

Missenard O, Mottet D, Perrey S (2008) Muscular fatigue increases signal-dependent noise during isometric force production. Neurosci Lett 437:154-157

Missenard O, Mottet D, Perrey S (2009) Factors responsible for force steadiness impairment with fatigue. Muscle Nerve 40:1019-1032

Moorhouse KM, Granata KP (2007) Role of reflex dynamics in spinal stability: intrinsic muscle stiffness alone is insufficient for stability. J Biomech 40:1058-1065 
Nesser TW, Huxell KC, Tincher JL, Okada T (2008) The relationship between core stability and performance in division I football players. J Srength Cond Res 22:1750-1754

Otten E (1999) Balancing on a narrow ridge: biomechanics and control. Phil Trans R Soc London B Biol Sci 354:869-875

Panjabi MM (1992) The stabilizing system of the spine. Part I. Function, dysfunction, adaptation, and enhancement. J Spinal Disord 5:383-389

Perrey S, Racinais S, Saimouaa K, Girard O (2010) Neural and muscular adjustments following repeated running sprints. Eur $\mathbf{J}$ Appl Physiol 109:1027-1036

Radebold A, Cholewicki J, Polzhofer GK, Greene HS (2001) Impaired postural control of the lumbar spine is associated with delayed muscle response times in patients with chronic idiopathic low back pain. Spine 26:724-730

Reeves NP, Everding VQ, Cholewicki J, Morrisette DC (2006) The effects of trunk stiffness on postural control during unstable seated balance. Exp Brain Res 174:694-700

Reeves NP, Cholewicki J, Narendra KS (2009) Effects of reflex delays on postural control during unstable seated balance. J Biomech 42:164-170
Selen LPJ, Beek PJ, Dieën JHv (2005) Can co-activation reduce kinematic variability? A simulation study. Biol Cybern 93:373-381

Seroussi RE, Pope ME (1987) The relationship between trunk muscle electromyography and lifting moments in the sagittal and frontal planes. J Biomech 20:135-146

Stokes IAF, Gardner-Morse M (2003) Spinal stiffness increases with axial load: another stabilizing consequence of muscle action. J Electromyogr Kinesiol 13:397-402

Taimela S, Kankaanpaa M, Luoto S (1999) The effect of lumbar fatigue on the ability to sense a change in lumbar position-a controlled study. Spine 24:1322-1327

Zazulak BT, Hewett TE, Reeves NP, Goldberg B, Cholewicki J (2007) Deficits in neuromuscular control of the trunk predict knee injury risk - a prospective biomechanical-epidemiologic study. Am J Sports Med 35:1123-1130

Zhang LQ, Rymer WZ (2001) Reflex and intrinsic changes induced by fatigue of human elbow extensor muscles. J Neurophysiol 86:1086-1194 